\title{
Estudo Técnico, Econômico e Ambiental da geração de energia através do biogás de lixo - o caso do aterro sanitário de Gramacho
}

\section{Technical, economical and enviromental study of energy production through biogas from waste - the case of Gramacho's landfill}

Artigo

Original

\author{
Fábio Viana de Abreu ${ }^{1}$ \\ Mila Rosendal Avelino ${ }^{2}$ \\ Diego Preza Monâco ${ }^{3}$
}

\section{Original}

Paper

\author{
Palavras-chave: \\ Biogás \\ Aterros Sanitários \\ Mecanismo de \\ Desenvolvimento \\ Limpo (MDL)
}

Resumo

A geração de energia através do biogás do lixo em aterros sanitários é uma alternativa para produção energia elétrica limpa, visando à redução dos impactos ambientais globais gerados pela queima dos resíduos sólidos urbanos. Neste trabalho, são definidas as condições operacionais do biogás, além do estudo e análise das áreas mais adequadas para o projeto e a vazão mínima de biogás, $\mathrm{em} \mathrm{m}^{3} / \mathrm{h}$, para viabilizar o mesmo. A contribuição ambiental mais relevante é a redução de emissões dos gases de efeito estufa (GEE), por meio da conversão do metano gerado em dióxido de carbono. De acordo com o Mecanismo de Energia elétrica Desenvolvimento Limpo (MDL), os países denominados desenvolvidos podem comprar créditos de carbono dos países em desenvolvimento (que possuam projetos verdes) para cumprir suas metas e objetivos ambientais. Essa alternativa de obtenção de receitas é uma linha de pesquisa deste estudo. São analisadas as tecnologias de conversão energética, com o estudo da melhor alternativa para a conversão energética do biogás de aterros sanitários. São realizados estudos comparativos, análises de sensibilidades e os resultados demonstraram que os grupos geradores, com o uso de motores a combustão interna (ciclos Otto ou Diesel), são mais viáveis no viés técnico e econômico para conversão energética do biogás de aterros sanitários no Brasil através de Termoelétricas a biogás.

\begin{abstract}
The generation of energy through biogas from waste in landfill is a way to produce clean electric energy, searching to reduce the global impacts generated by the burning of the urban solid wastes (USW). In this paper the operational conditions of biogas are defined, and the study and analysis of the appropriated areas to the project and the minimum flow of biogas in $\mathrm{m}^{3} / \mathrm{h}$, to enable this project. The most significant environmental contribution is the reduction of the emissions of greenhouse gases (GEE) by the conversion of the methane in carbon dioxide. In accordance to the Clean Development Mechanism (CDM), the developed countries can acquire carbon credits from the in development countries (the ones that possess green projects) in order to reach their goals and environmental aims. The alternative of obtaining incomes is the research line in this study. The technologies of energy conversion are analyzed altogether with the study of the best alternative to the energetic conversion of biogas in the landfill. Comparative studies and sensitivity analysis are done and the results showed that the generating groups, using motor of internal combustion (cycles Otto or Diesel), are more able in a technical and economical part to the biogas energy conversion in the landfills in Brazil through biogas thermoelectric.
\end{abstract}

Key words:

Biogas

Landfill

Urban Solid Waste

Clean Development Mechanism (CDM)

Eletrical Energy
Recebido em 04/2011

Aprovado em 08/2011 
aterro sanitário deverá receber no mínimo 200 toneladas/dia de resíduos, com capacidade mí-

A geração de desperdício e excessos no Brasil são reflexos da adoção de um desenvolvimento com a característica de alto padrão consumista. Por isso, a busca de soluções adequadas para a disposição final dos resíduos sólidos de forma integrada, da sua origem até a disposição final, é essencial para o desenvolvimento sustentável [Abreu, 2009].

A gestão adequada do lixo e a geração de energia através do biogás de lixo em aterros sanitários são soluções ambientalmente sustentáveis (gerando energia elétrica renovável e limpa). Além disso, o estudo da geração de energia elétrica a partir do biogás permite a redução de fugas dos gases de efeito estufa (GEE) e a maximização do índice de conversão do metano, contabilizado no cálculo para emissão de créditos de carbono dentro do mecanismo de desenvolvimento limpo (MDL) [Abreu, 2009].

A negociação de créditos de carbono é a forma transacional do MDL [UNFCCC, 2007]. Tais iniciativas induzem investimentos em projetos sustentáveis em que pode haver redução de emissões e/ou sequestro de carbono, assegurando um modelo de desenvolvimento limpo para os países emergentes, onde os custos de implementação de tais projetos são maiores [Cebds, 2001]. O Brasil, enquanto país signatário do Tratado de Kyoto, está habilitado a desenvolver projetos de redução dos GEE e emitir os créditos aos países industrializados que devam reduzir suas emissões até o ano 2012.

O biogás gerado nos aterros sanitários é composto basicamente por metano $\left(\mathrm{CH}_{4}-\mathrm{de}\right.$ 55 a $65 \%)$, dióxido de carbono $\left(\mathrm{CO}_{2}-\right.$ de 30 a $40 \%$ ), nitrogênio $\left(\mathrm{N}_{2}-\right.$ de 0 a $\left.1 \%\right)$, hidrogênio $\left(\mathrm{H}_{2}-\right.$ de 0 a $\left.2 \%\right)$ e gás sulfídrico $\left(\mathrm{H}_{2} \mathrm{~S}-\right.$ de 0 a 1\%) [Polprasert, 1996]. Em um período de 100 anos, 1 grama de metano contribui 21 vezes mais para a formação do efeito estufa do que 1 grama de dióxido de carbono [UNFCCC, 2007]. A combustão completa do metano produz dióxido de carbono e vapor d'água.

A geração de biogás em um aterro sanitário é iniciada algumas semanas após o início do depósito dos resíduos e continua por 15 anos após seu encerramento. Uma tonelada de resíduo disposto em um aterro sanitário gera em média $200 \mathrm{Nm}^{3}$ de biogás. Para comercializar o biogás, através da recuperação energética, o nima de recepção da ordem de 500.000 toneladas em sua vida útil e altura mínima de carregamento de 10 metros [World Bank, 2005].

$\mathrm{O}$ aterro de Gramacho, situado na cidade de Duque de Caxias (RJ), foi escolhido como o estudo de caso. Originalmente, era um lixão que, a partir do início dos anos da década de 1990, passou a receber alguns cuidados para minimizar a agressão que causava ao meio ambiente. O mais recente foi a conclusão da primeira fase da Estação de Tratamento de Efluentes Líquidos, que trata diariamente, segundo a Comlurb [Comlurb, 2009], 960 metros cúbicos de chorume, que era uma das principais preocupações dos ambientalistas os quais temiam a contaminação da Baía de Guanabara. O próximo passo é a produção de energia através do biogás do lixo.

Atualmente, a cidade do Rio de Janeiro tem a sua produção de 8 a 9,3 mil ton./dia de lixo. Considerando o valor de 263.370 toneladas/mês, pode-se concluir que a produção diária da cidade do Rio de Janeiro encontra-se em aproximadamente no valor de 8.779 toneladas/dia (Araruna, 2008).

De acordo com Araruna (2008), “a coleta e a destinação de resíduos sólidos comprometem de $7 \%$ a $15 \%$ dos orçamentos municipais". Como as prefeituras costumam gastar cerca de $60 \%$ de seus recursos com folha de pagamento, o lixo muitas vezes lidera a lista de outras despesas. Com isso, destaca-se a importância de estudos técnicos e científicos que resultem em soluções e/ou alternativas para minimizar o problema dos resíduos sólidos urbanos.

\section{Materiais e Métodos}

$\mathrm{O}$ aterro de Gramacho é o estudo de caso escolhido, devido à sua importância para a cidade do Rio de Janeiro e seu entorno.

Para a realização do EVTE, foram levantados os dados técnicos para estudo das alternativas de geração de eletricidade a partir de biogás, bem como a determinação do potencial de biogás a ser produzido e do potencial de eletricidade a ser gerado. Para isso, o modelo utilizado foi o recomendado pela Agência de Proteção Ambiental dos Estados Unidos (USEPA) [EPA, 2005]. 
A estimativa de produção de metano está expressa na Eq. (1):

$$
\mathrm{Q}_{\mathrm{M}}=\sum_{\mathrm{i}=1}^{\mathrm{n}} 2 \mathrm{k} \mathrm{L}_{0} \mathrm{Mi}\left(\mathrm{e}^{-\mathrm{kti}}\right)
$$

O modelo da USEPA requer o conhecimento do histórico da deposição dos resíduos (ou, no mínimo, da quantidade de lixo depositado e da data de abertura do aterro sanitário); usa uma função exponencial de degradação de primeira ordem, que presume que a geração de biogás alcance o ponto máximo após um intervalo de tempo que representa o período antes da geração de metano. O modelo da USEPA supõe um intervalo de um ano entre a deposição dos resíduos e a geração de biogás. Após um ano, o modelo prevê que a geração de biogás decresça exponencialmente à medida que é consumida a fração orgânica dos resíduos.

Os dados de quantidade de lixo anual são inseridos e os parâmetros de velocidade de degradação $(\mathrm{k}=0,060)$ e potencial de metano $\left(\mathrm{L}_{0}\right.$ $=84,6$ ) são adotados de acordo com as condições da região estudada. E para a realização da análise econômica utilizou-se os parâmetros Valor Presente Líquido (VPL) e Taxa Interna de Retorno (TIR).

\subsection{Valor Presente Líquido (VPL)}

É o somatório dos custos e das receitas líquidas do projeto (ou previstas) durante sua vida econômica, trazidos a data de atualização do fluxo de caixa, utilizando-se para isso a taxa de desconto apropriada: a taxa mínima de atratividade - TMA - do segmento de negócio. A fórmula do VPL é definida pela Eq. (2), como:

$$
\mathrm{VPL}=\sum_{K=0}^{n} \frac{E\left(C F_{K}\right)}{(1+i)^{k+j}}
$$

Onde:

E Valor esperado;

CF Valor genérico do fluxo de caixa liquido no período $\mathrm{k}$ (positivo se for beneficio, negativo se for custo);

i Taxa de desconto: TMA;

k Período no instante $\mathrm{k}$ do fluxo de caixa;

j Posição do vetor no período (inicio $=0$ )

\subsection{Taxa Interna de Retorno (TIR)}

A taxa interna de retorno considera os desembolsos da empresa - valores negativos - para fazer um projeto como um "empréstimo" a ser "pago" posteriormente com os embolsos ou receitas - valores positivos - proporcionados pelo mesmo projeto. Com essa metodologia, a TIR é a taxa de retorno implícita no fluxo de caixa, que só depende da relação entre os valores positivos e negativos, a qual iguala esses desembolsos aos embolsos com isso tornando o $\mathrm{VPL}=0$.

A TIR é uma taxa média que considera toda a vida econômica do projeto e é expressa em termos anuais. Esta taxa é calculada obtendo-se as raízes da Eq. (3):

$$
\text { TR: } \sum_{K=0}^{n} \frac{E\left(C F_{K}\right)}{(1+T I R)^{k+j}}=0
$$

\subsection{Tributos}

Para a elaboração do Estudo de Viabilidade Técnica e Econômica do projeto é essencial o estudo e análise dos tributos incidentes nas receitas provenientes da venda de energia elétrica produzida e créditos de carbono. De acordo com a Tab. (1) (MME, 2005), serão apresentados os tributos que serão incluídos no fluxo de caixa.

Tabela 1: Tributos incidentes em projetos de energia

\begin{tabular}{|c|c|c|c|c|}
\hline \multicolumn{5}{|c|}{ Tributos } \\
\hline & Tributos & Alíquota & Incidência & Competência \\
\hline 1 & COFINS & $3,0 \%$ & Receitura Bruta & FEDERAL \\
\hline 2 & PIS & $0,65 \%$ & Receitura Bruta & FEDERAL \\
\hline 3 & ICMS & $0 \%$ & Receitura Bruta & ESTADUAL \\
\hline 4 & IR* & $15+10 \%$ & Lucro antes dos impostos & FEDERAL \\
\hline 5 & CSLL & $9 \%$ & Lucro antes dos impostos & FEDERAL \\
\hline
\end{tabular}

$\mathrm{IR}^{*}=15 \%$ de até $\mathrm{R} \$ 240.000 /$ ano $+10 \%$ acima de $\mathrm{R} \$ 240.000 /$ ano 


\section{Resultados}

Com a aplicação da metodologia USEPA no aterro de Gramacho, sua recuperação de biogás de lixo em 2009, foi de $30.000 \mathrm{~m}^{3} / \mathrm{h}$. Após o fechamento do local, estima-se hoje que a recuperação do gás de lixo venha a se declinar rapidamente, conforme o Fig. (1).

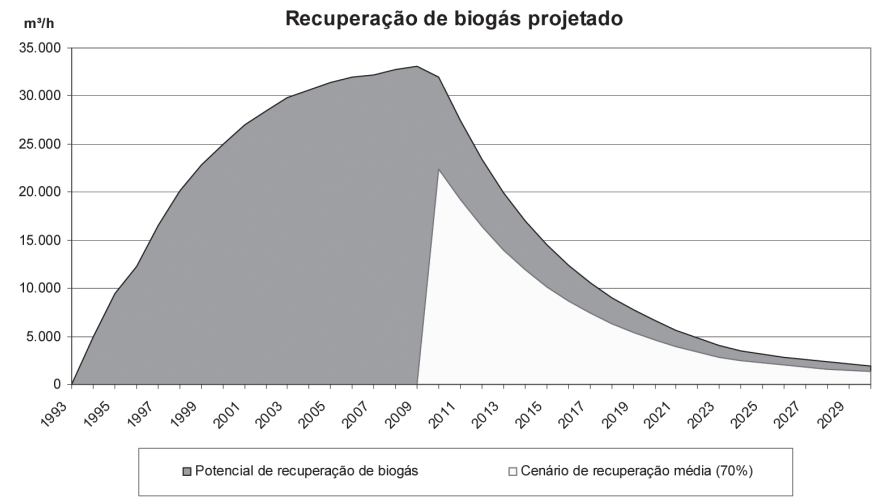

Figura 1: Recuperação projetada de biogás no Aterro Sanitário de Gramacho

Na Tab. (2) é apresentado um sumário das taxas de recuperação de biogás e capacidade (Bruta) da Usina Termoelétrica (UTE).

\begin{tabular}{|c|c|c|}
\hline Ano & $\begin{array}{c}\text { Taxa de Recuperação de Biogás } \\
\text { projetada }\left(\mathbf{m}^{\mathbf{3}} \mathbf{h} \mathbf{h}\right)\end{array}$ & $\begin{array}{c}\text { Capacidade Bruta da Usina de } \\
\text { Motor de Combustão Interna }(\mathbf{k W})\end{array}$ \\
\hline 2012 & 23.404 & 10.031 \\
\hline 2013 & 19.973 & 10.031 \\
\hline 2014 & 17.045 & 10.031 \\
\hline 2015 & 14.546 & 10.031 \\
\hline 2016 & 12.414 & 10.031 \\
\hline 2017 & 10.594 & 10.031 \\
\hline 2018 & 9.041 & 7.165 \\
\hline 2019 & 7.716 & 7.165 \\
\hline 2020 & 6.585 & 7.165 \\
\hline 2021 & 5.619 & 4.299 \\
\hline 2022 & 4.796 & 4.299 \\
\hline 2023 & 4.093 & 4.299 \\
\hline 2024 & 3.493 & 4.299 \\
\hline
\end{tabular}

Tabela 2: Sumário das Taxas de Recuperação de Biogás e Capacidade (Bruta) da Usina Termoelétrica

\section{Discussão}

Das tecnologias convencionais para a conversão energética do biogás, destacam-se as turbinas a gás e os motores de combustão interna (ciclos Otto e Diesel). Para geração de energia a capacidades pequenas e médias, os motores a combustão interna são mais adequados devido ao seu menor custo e maior eficiência nesta faixa. Somente para altas capacidades, as turbinas a gás passam a ter economicidade, melhorada quando utilizadas em ciclos combinados. Os motores a combustão interna de ciclos Otto ou Diesel possuem maior eficiência na faixa de operação deste projeto. Motores de ciclo Diesel trabalham com taxas de compressão mais elevadas, sendo necessário operar nestes com o biogás misturado ao diesel ou biodiesel; o que representaria um insumo adicional para o Aterro Sanitário.

A seguir, é apresentada na Tab. (3), a comparação da potência e rendimento para as tecnologias de conversão energética Motor a Combustão Interna e Turbinas. 
Tabela 3: Tecnologias de Conversão

\begin{tabular}{|c|c|c|}
\hline Tecnologia de Conversão & Potência Instalada & Rendimento Elétrico \\
\hline Motores a Gás (Ciclo Otto) & $30 \mathrm{~kW}-1 \mathrm{MW}$ & $25 \%-30 \%$ \\
\hline Motores a Gás (Ciclo Diesel) & $40 \mathrm{~kW}-20 \mathrm{MW}$ & $30 \%-40 \%$ \\
\hline Tubina a Gás (Médio Porte) & $500 \mathrm{~kW}-150 \mathrm{MW}$ & $35-42 \%$ \\
\hline
\end{tabular}

A Tab. (4) mostra um sumário dos resultados da avaliação econômica no cenário com tributos da UTE, apresentando o Valor Presente Líquido (VPL) e a Taxa Interna de
Retorno (TIR) do projeto, realizando uma análise de sensibilidade, utilizando valores diversos as receitas oriundas das taxas de créditos de carbono (CER).

Tabela 4: Sumário da Avaliação Econômica com UTE e tributos

\begin{tabular}{|c|c|c|c|}
\hline \multicolumn{4}{|c|}{ Investimento inicial de 100\% $=\mathbf{1 5 . 5 1 4 . 8 8 0}$} \\
\hline $\begin{array}{c}\text { CER } \\
\left(\text { US\$ / ton. } \text { CO }_{2 \text { eq }} \text { ) }\right.\end{array}$ & TIR & VPL & $\begin{array}{c}\text { TAXA DE VENDAS FORA } \\
\text { DO LOCAL (\$/KWh) }\end{array}$ \\
\hline 10 & $14,71 \%$ & $\$ 8.880 .212$ & 0,045 \\
\hline 10 & $15,96 \%$ & $\$ 11.476 .556$ & 0,055 \\
\hline 10 & $17,07 \%$ & $\$ 14.072 .901$ & 0,065 \\
\hline 10 & $18,06 \%$ & $\$ 16.669 .246$ & 0,075 \\
\hline
\end{tabular}

\begin{tabular}{|c|c|c|c|}
\hline \multicolumn{4}{|c|}{ Investimento inicial de 25\% $=\mathbf{3 . 8 7 8 . 7 2 0}$} \\
\hline $\begin{array}{c}\text { CER } \\
\left(\text { US\$ / ton. } \text { CO }_{2 \text { eq }} \text { ) }\right.\end{array}$ & TIR & VPL & $\begin{array}{c}\text { TAXA DE VENDAS FORA } \\
\text { DO LOCAL (\$/KWh) }\end{array}$ \\
\hline 10 & $20,95 \%$ & $\$ 10.129 .089$ & 0,045 \\
\hline 10 & $22,56 \%$ & $\$ 12.725 .434$ & 0,055 \\
\hline 10 & $23,96 \%$ & $\$ 15.321 .779$ & 0,065 \\
\hline 10 & $25,22 \%$ & $\$ 17.918 .123$ & 0,075 \\
\hline
\end{tabular}

\begin{tabular}{|c|c|c|c|}
\hline \multicolumn{4}{|c|}{ Investimento inicial de $100 \%=15.514 .880$} \\
\hline $\begin{array}{c}\text { CER } \\
\left(\mathrm{USS} / \text { ton. } \mathrm{CO}_{2 \mathrm{eq}}\right) \\
\end{array}$ & TIR & VPL & $\begin{array}{c}\text { TAXA DE VENDAS FORA } \\
\text { DO LOCAL }(\$ / K W h) \\
\end{array}$ \\
\hline 17 & $23,81 \%$ & $\$ 27.232 .359$ & 0,045 \\
\hline 17 & $24,47 \%$ & $\$ 29.828 .704$ & 0,055 \\
\hline 17 & $25,09 \%$ & $\$ 32.425 .049$ & 0,065 \\
\hline 17 & $25,67 \%$ & $\$ 35.021 .393$ & 0,075 \\
\hline
\end{tabular}

\begin{tabular}{|c|c|c|c|}
\hline \multicolumn{4}{|c|}{ Investimento inicial de 25\% $=\mathbf{3 . 8 7 8 . 7 2 0}$} \\
\hline $\begin{array}{c}\text { CER } \\
\text { (US\$ / ton. } \text { CO }_{2 \text { eq }} \text { ) }\end{array}$ & TIR & VPL & $\begin{array}{c}\text { TAXA DE VENDAS FORA } \\
\text { DO LOCAL (\$/KWh) }\end{array}$ \\
\hline 17 & $34,31 \%$ & $\$ 28.481 .237$ & 0,045 \\
\hline 17 & $35,05 \%$ & $\$ 31.077 .582$ & 0,055 \\
\hline 17 & $35,75 \%$ & $\$ 33.673 .926$ & 0,065 \\
\hline 17 & $36,42 \%$ & $\$ 36.270 .271$ & 0,075 \\
\hline
\end{tabular}

Com isso, cenários com os créditos de carbono a U.S. $\$ 10$ ton. $\mathrm{CO}_{2}$ eq. e o valor da energia elétrica no patamar de no mínimo U.S. $\$ / \mathrm{kWh} 0,045$ o projeto também é viável, mesmo com a inclusão dos tributos.

\section{Conclusão}

O presente trabalho investigou as condições de produção e viabilidade técnica e econômica de biogás de lixo em aterros sanitários, 
com a escolha do aterro de Gramacho para o estudo. Com isso, foi proposta uma destinação final de RSU's mais adequação, proporcionando a redução do chorume, dos odores e da poluição ambiental. Como consequência, elevou-se a vida útil do aterro sanitário e segurança ambiental local.

A produção de energia através do biogás do lixo em aterros sanitários representa ganhos para a sociedade (geração de empregos e redução de subempregos), para as prefeituras (representam uma fonte extra de renda com a comercialização da energia gerada pelo biogás) e para o meio ambiente com a contenção de emissões de $\mathrm{CH}_{4}$, redução do uso de combustíveis fósseis, no caso de aproveitamento energético, redução de odores e vetores nos aterros devido a boas práticas de gerenciamento, dentre outras (Abreu, 2009).

Para a cidade do Rio de Janeiro, onde os resíduos sólidos urbanos representam uma despesa de cerca de $6 \%$ a $8 \%$ do orçamento público anual, uma Usina a Biogás seria uma oportunidade de transformar um passivo contábil, financeiro e ambiental em receitas para município de acordo com as taxas de VPL e TIR calculadas para cada hipótese da análise financeira.

Com a produção de $10 \mathrm{MW}$ de energia no início do projeto, seria possível atender às necessidades energéticas da própria Usina de Biogás e mais uma parcela da população fluminense, de acordo com o perfil do consumo energético das famílias atendidas.

Realizando um estudo da matriz energética nacional para o setor, constata-se que conforme a Cetesb (1999), a geração de metano em depósitos de resíduos sólidos urbanos brasileiros está na ordem de $677 \mathrm{Gg}$., cuja densidade padrão definida é de $0,716 \mathrm{Kg} / \mathrm{m}^{3}$, o que significa cerca de 945 milhões de metros cúbicos anuais. Como o metano representa cerca de 50 a $55 \%$ do volume de biogás, têm-se 1.718 milhões de metros cúbicos anuais desse gás, considerando uma recuperação típica de $90 \%$, sendo que estariam disponíveis $1.546 \mathrm{Mm}^{3}$ de biogás para geração de energia elétrica. Isso representaria ao País, utilizando usinas termoelétricas baseadas em motores a combustão interna com eficiência na faixa de $20 \%$ (caso do ciclo Otto), uma energia disponível de 2,1TWh, que alimentaria 1750 mil residências com o consumo médio mensal de $100 \mathrm{kWh}$, o que equivale a uma ci- dade de mais de 3 milhões de habitantes. Como muitos aterros não têm economicidade para produção de energia através do biogás de lixo, a energia disponível seria um patamar ainda bem menor que os 2,1 TWh.

Diante do exposto, é possível constatar que o biogás não tem um impacto significativo na matriz brasileira mesmo considerando o valor de 2,1 TWh com relação a oferta total de 497,4 TWh que não representa nem 1\% da matriz energética brasileira. Mas se todas as cidades brasileiras utilizassem o biogás de lixo em detrimento de outras fontes de energias mais poluentes, seria possível contribuir para redução das consequências das mudanças climáticas visto que o gás metano é mais nocivo que o gás carbônico ( $\left.\mathrm{CO}_{2}\right)$. Outra vantagem do biogás de lixo com relação às hidroelétricas seria sua utilização para gerar energia elétrica próximo ao centro consumidor, evitando assim investimentos em novas linhas de transmissão de energia elétrica.

Por fim, a implantação de uma termelétrica a partir do biogás do lixo, gera diversas contribuições para o País (principalmente para a região atendida), dentre as quais, serão listadas as principais abaixo:

a. contribuição para a sustentabilidade ambiental local;

b. contribuição para o desenvolvimento das condições de trabalho e a geração líquida de empregos;

c. contribuição para a distribuição de renda;

d. contribuição para capacitação e desenvolvimento tecnológico (possibilidade de reprodução da tecnologia empregada, observando o seu efeito demonstrativo, avaliando, ainda, a origem dos equipamentos, a existência de royalties e de licenças tecnológicas e a necessidade de assistência técnica internacional);

e. contribuição para o desenvolvimento regional, que pode ser medida a partir da integração do projeto com outras atividades sócioeconômicas na região de sua implantação.

f. conscientização da população com relação ao desenvolvimento de práticas sustentável e produção de energia renovável e limpa (Abreu, 2009). 


\section{Agradecimentos}

Ao CNPQ, Conselho Nacional de Desenvolvimento Científico e Tecnológico e a Universidade do Estado do Rio de Janeiro

\section{Referências}

1. ABREU, F. V.; COSta Filho, M. A. F.; SOUZA, M. C. L.. Technical and economical feasibility analysis of energy generation though the biogas from garbage in landfill - an alternative of renewable energy generation. In: 20th International Congress of Mechanical Engineering (COBEM), Gramado - RS, 2009.

2. ABREU, F. V.; COSTA FILHO, M. A. F.; SOUZA, M. C. L.. Biogás de aterros sanitários para geração de energia renovável e limpa - um estudo de viabilidade técnica e econômica. In: IX Congreso Iberoamericano de Ingeniería Mecânica (CIBIM), 2009.

3. ABREU, F. V.; Dissertação de Mestrado, "Análise de Viabilidade Técnica e Econômica da Geração de Energia Através do Biogás de Lixo em Aterros Sanitários", UERJ - Universidade do Estado do Rio de Janeiro - 2009

4. ABREU, F. V.; $<$ http:// municipalsolidwaste.blogspot.com/> acessado em Abril de 2011.

5. ABREU, F. V.; <http://biogasdelixo. blogspot.com/> acessado em Abril de 2011.

6. ARARUNA, José - Reportagem sobre Resíduos Sólidos Urbanos com Prof. José Araruna, pag. 20 - Jornal O Globo, 2008.

7. COMLURB: Companhia Municipal de Limpeza Urbana <http://comlurb.rio. rj.gov.br/etc_atgramacho.htm>, acessado em 20 de maio de 2010.

8. DUARTE, Adriana Carneiro; Braga, Maria Cristina Borba - Brazilian projects of MDL in Landfill. $24^{\circ}$ Brazilian congress of Sanitary and Ambient Engineers, 2007.
9. EPA, 2005. Landfill Gas Emissions Model (LandGEM) Version 3.02 User's Guide. EPA-600/R05/047 (May 2005), Research Triangle Park, NC. U.S. Environmental Protection Agency.

10. FERREIRA, J.A. Solid Wast: Current perspectives In: Sisinno, C.L.S. \& Oliveira, R.M de (Org.). Solid residues, Environment and Health: a vision to multidiscipline. Rio de Janeiro: Fiocruz, 2000. p. 19-40.

11. LORA, E. E. S.; Nascimento, M. A. R. coord. Thermoelectric generation: Planning, Project and Operation, 2004, 457 - 461 pp.

12. POLPRASERT, C. Organic Waste Recyclin Technology And Management. 2nd Edition. John Wiley \& Sons. 412 P. Chichester, 1996.

13. SCS Engineers, 2005. <http://www. bancomundial.org.ar/lfg/archivos/ PrefeasibilityStudies/Spanish_Portuguese/ Gramacho_PreFeasibility_Study_Portuguese. pdf $>$, access in the 15 of mar 2009.

14. UN. United Nations. Conference on Environment and Development - 1992.

15. UN. United Nations. Kyoto Protocol to The United Nations Framework Convention On Climate Change. United Nations. 21 P. Washington - Dc, 2002.

16. UNFCCC. United Nations Framework Convention on Climate Change. Kyoto Protocol Http://Unfecc.Int/2860.Php Access in the 26 Nov. 2007a.

17. UNFCCC. United Nations Framework Convention on Climate Change. Overview of Project Activity Cycle. $<\mathrm{Http}: / /$ Unfccc. Int/2860.Php > Access in the 25 Nov. $2007 b$.

18. World Bank. Guidance Note on Recuperation of Landfill Gas From Municipal Solid Waste Landfills, 2005.

Endereço para Correspondência:

Fábio Viana de Abreu

fabio.viana.abreu@gmail.com

Rua Gonzaga Bastos, n 131, apto 504

Vila Isabel - Rio de Janeiro

CEP: 20541-000 